\title{
Mass insertions vs. mass eigenstates calculations in flavour physics
}

\author{
A. Dedes, ${ }^{a, b}$ M. Paraskevas, ${ }^{a}$ J. Rosiek, ${ }^{c}$ K. Suxho ${ }^{a}$ and K. Tamvakis ${ }^{a}$ \\ ${ }^{a}$ Department of Physics, Division of Theoretical Physics, \\ University of Ioannina, GR 45110, Greece \\ ${ }^{b}$ University of Athens, Physics Department, \\ Nuclear and Particle Physics section, GR 15771 Athens, Greece \\ ${ }^{c}$ Institute of Theoretical Physics, Physics Department, \\ Warsaw University, Pasteura 5, 02-093 Warsaw, Poland \\ E-mail: adedes@cc.uoi.gr, mparask@grads.uoi.gr, \\ janusz.rosiek@fuw.edu.pl, csoutzio@cc.uoi.gr, tamvakis@uoi.gr
}

ABSTRACT: We present and prove a theorem of matrix analysis, the Flavour Expansion Theorem (or FET), according to which, an analytic function of a Hermitian matrix can be expanded polynomially in terms of its off-diagonal elements with coefficients being the divided differences of the analytic function and arguments the diagonal elements of the Hermitian matrix. The theorem is applicable in case of flavour changing amplitudes. At one-loop level this procedure is particularly natural due to the observation that every loop function in the Passarino-Veltman basis can be recursively expressed in terms of divided differences. FET helps to algebraically translate an amplitude written in mass eigenbasis into flavour mass insertions, without performing diagrammatic calculations in flavour basis. As a non-trivial application of FET up to a third order, we demonstrate its use in calculating strong bounds on the real parts of flavour changing mass insertions in the up- squark sector of the MSSM from neutron Electric Dipole Moment (nEDM) measurements, assuming that $\mathrm{CP}$-violation arises only from the CKM matrix.

KEYWORDS: Supersymmetry Phenomenology

ARXIV EPRINT: 1504.00960v2 


\section{Contents}

1 Introduction 1

2 A warming-up example: flavour calculation techniques 3

3 Flavour expansion theorem $\quad \mathbf{5}$

3.1 Hermitian matrix function and divided differences 6

3.2 The Flavour Expansion Theorem (FET) 7

$\begin{array}{lll}3.3 & \text { Divided differences and Passarino-Veltman functions } & 7\end{array}$

3.4 Applications and limitations of FET 8

4 Expansion of fermionic amplitudes $\quad 9$

5 Application of FET: neutron EDM in the MSSM 12

$\begin{array}{llr}6 & \text { Summary } & 16\end{array}$

$\begin{array}{ll}\text { A Proof of the Flavour Expansion Theorem } & 17\end{array}$

$\begin{array}{ll}\text { A.1 Fully symmetrized polynomials } & 17\end{array}$

$\begin{array}{lll}\text { A.2 Flavour Expansion Theorem: the proof } & 18\end{array}$

B Convergence criterion for FET expansion of the one-loop functions 20

\section{Introduction}

Within the general framework of a perturbative Quantum Field Theory (QFT), the standard strategy followed when calculating physical transition amplitudes, is to express the Lagrangian density in a particular field basis, commonly referred to as mass eigenstate basis. Contrary to any other possible choice, only in this case the states of the theory correspond to physical particles with definite mass and symmetry charges. Up to possible mass degeneracies, this basis is unique and is characterized by the absence of quadratic mixing terms between different mass eigenstates. Furthermore, all parameters of the Lagrangian in this basis, are physically observable, in the sense that all masses and couplings can in principle be obtained by a suitable experiment. After having set the Lagrangian to the mass eigenstates fields basis, one can then deploy the standard QFT machinery and set the Feynman rules in order to calculate transition amplitudes for any physical process.

Nevertheless, in the vast majority of the models we are interested in, masses are typically generated or affected by a symmetry breaking mechanism. In this case another basis is physically meaningful as well. This is the basis where the Lagrangian exhibits explicitly the properties of the initial symmetry, and the states correspond to eigenstates of a larger 
symmetry group. We will refer to these eigenstates, for gauge bosons and collectively for fermions and scalars with family replication, as gauge and flavour eigenstates respectively, although in our definition for the latter there is no implicit requirement of an underlying flavour symmetry. In this sense the flavour eigenstate basis in many models can be considered in practice arbitrary, constrained only by the other symmetries of the initial Lagrangian, i.e., gauge symmetry, supersymmetry, etc. The transformation from the initial basis to the mass eigenstate basis, which is still the physical basis of the theory, is performed with mass diagonalization involving unitary transformations and field redefinitions, typically leaving a physical imprint on the parameters of the mass eigenstate theory. In the Standard Model (SM) [1-3] this effect is displayed in the gauge sector through the weak mixing angle and in the fermion sector through the CKM $[4,5]$ and PMNS $[6,7]$ matrices of charged currents. However, even in this very successful model, the CKM or PMNS parameters along with the fermion mass eigenvalues are insufficient to determine unambiguously the flavour eigenstate basis.

Although the mass eigenstate basis of a perturbative QFT is the natural basis for calculations of physical processes, some effects typically related to the symmetries of the Lagrangian before symmetry breaking are better understood in flavour basis. ${ }^{1}$ Therefore for a qualitative analysis of such effects, it is often useful to have our expressions in the latter basis. ${ }^{2}$ The standard strategy that has been employed up to date, is an approximate diagrammatic method commonly referred to as the Mass Insertion Approximation (MIA) [8-10]. In this approach one defines the Feynman rules of the theory directly in flavour basis. The diagonal part of the flavour mass matrix is typically absorbed into the definition of (unphysical) massive propagators and the non-diagonal part commonly referred to as mass insertions is treated perturbatively, as part of the interaction Lagrangian which now possesses quadratic mixing terms. Due to the presence of quadratic interactions, besides the standard loop approximation of a perturbative QFT, there is an extra approximation for each diagram, appearing as an infinite series in terms of mass insertions, following the presence of a flavour propagator.

In what follows, we present an algebraic treatment of transition amplitudes in mass eigenstate basis, leading directly to the corresponding amplitudes in flavour basis, in the form of the MIA or of an equivalent expansion. In particular, we prove a theorem in matrix analysis [11, 12], that we coin Flavour Expansion Theorem or simply FET, which says that an analytic function of a Hermitian matrix can be expanded polynomially in terms of its offdiagonal elements with coefficients being the divided difference of the analytic function and arguments the diagonal elements of the Hermitian matrix. At one-loop level, this expansion is naturally related to the remarkable recursive properties of next order Passarino-Veltman (PV) function [13] being the divided difference [14] of the previous one. We then argue that FET connects mass and flavour field bases amplitudes. The first non-trivial order

\footnotetext{
${ }^{1}$ Since in many cases the mass diagonalization of various sectors of the theory is independent of each other, one can also work in a mixed basis where some sectors are given in mass basis and others in flavour basis. In what follows, the basis we work can be easily identified from the context.

${ }^{2}$ This is after all the basis that more naturally connects couplings and masses to high energies through their Renormalization Group Equations (RGEs).
} 
in the expansion [cf. eq. (3.11)], and applications in MSSM flavour physics, have been presented in refs. $[15,16]$ but a formal mathematical proof to all orders was unknown until now. FET is especially useful when is used to evaluate higher order expansion terms, it is technically easier, elegant and superior to often tedious, time-consuming and thus prone to mistakes calculations of the diagrammatic MIA. We support our claims with a novel example towards the end of the article.

More specifically, the paper is organized as follows: in section 2, we present a warmingup example of a scalar toy-model in order to illustrate the relation between the calculation of flavour transition amplitudes in mass and flavour bases. Then, in section 3, we formulate a general algebraic theorem dealing with the expansion of an analytic function of a Hermitian matrix, and, discuss its applications to rewriting flavour amplitudes with scalar and vector particles, from mass to flavour eigenstates basis. We extend our discussion to the case of amplitudes involving fermions in section 4. In section 5, we illustrate the developed technique on a (potentially) physical example, expanding the dominant gluinosquark contribution to the neutron Electric Dipole Moment and showing the importance of higher order terms. We conclude our results in section 6 . Finally, the formal proof of theorem formulated in section 3 is given in appendix A, while in appendix B we derive the convergence criterion for the mass insertion expansion of the one-loop integrals.

\section{A warming-up example: flavour calculation techniques}

To set up a simple framework to introduce the standard techniques of flavour physics calculations, we consider a scalar field toy model composed of $N$-complex charged scalar fields $\Phi_{I}$, with family replication, and an extra neutral, real, scalar field $\eta$. The (squared) mass matrix, $\mathbf{M}^{2}$, and the Yukawa coupling matrix, $\mathbf{Y}$, of the flavour eigenstates $\Phi_{I}$, are necessarily Hermitian but not aligned in general. The Lagrangian density, will have the form: ${ }^{3}$

$$
\mathcal{L}_{\text {flavour }}=\left(\partial^{\mu} \Phi_{I}^{\dagger}\right)\left(\partial_{\mu} \Phi_{I}\right)-M_{I J}^{2} \Phi_{I}^{\dagger} \Phi_{J}+\frac{1}{2}\left(\partial^{\mu} \eta\right)\left(\partial_{\mu} \eta\right)-\frac{1}{2} m_{\eta}^{2} \eta^{2}-Y_{I J} \eta \Phi_{I}^{\dagger} \Phi_{J}-\ldots
$$

where dots denote additional scalar field interactions which are irrelevant for the discussion below. Using the unitary rotation,

$$
\Phi_{I}=U_{I i} \phi_{i}
$$

where $\mathbf{U}$ satisfies the condition

$$
\mathbf{U}^{\dagger} \mathbf{M}^{\mathbf{2}} \mathbf{U}=\mathbf{m}^{\mathbf{2}}=\operatorname{diag}\left(m_{1}^{2}, \ldots, m_{N}^{2}\right),
$$

one can express the Lagrangian in terms of mass eigenstates $\phi_{i}$

$$
\mathcal{L}_{\text {mass }}=\left(\partial^{\mu} \phi_{i}^{\dagger}\right)\left(\partial_{\mu} \phi_{i}\right)-m_{i}^{2} \phi_{i}^{\dagger} \phi_{i}+\frac{1}{2}\left(\partial^{\mu} \eta\right)\left(\partial_{\mu} \eta\right)-\frac{1}{2} m_{\eta}^{2} \eta^{2}-y_{i j} \eta \phi_{i}^{\dagger} \phi_{j}+\ldots
$$

where the transformed scalar couplings are identified as $y_{i j}=U_{i I}^{\dagger} Y_{I J} U_{J j}$.

\footnotetext{
${ }^{3}$ Sum over repeated indices is always assumed in the text, unless stated otherwise.
} 


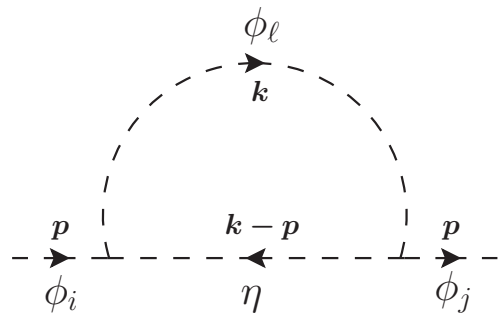

Figure 1. Scalar self-energy $-i \Sigma_{j i}(p)$ in the mass eigenstate basis.

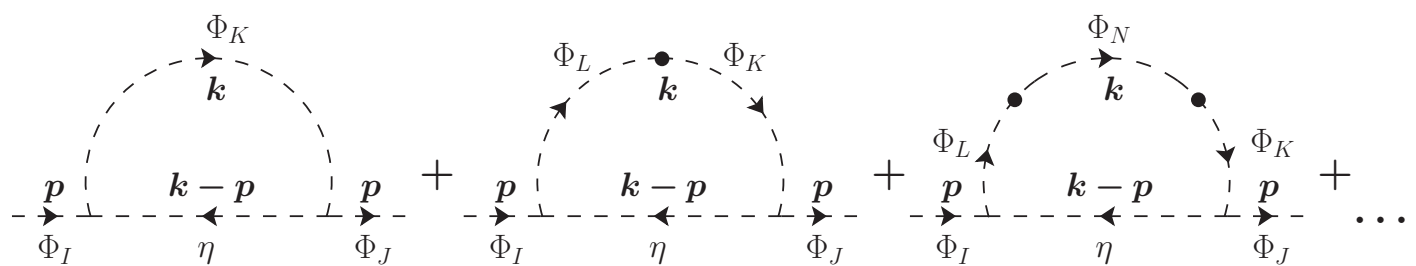

Figure 2. Scalar self-energy $-i \hat{\Sigma}_{J I}(p)$ in the flavour basis.

First, let us consider the "flavour" changing one-loop One-Particle-Irreducible (1PI) self-energy diagram of the mass eigenstates fields $\phi_{i}$, shown in figure 1 . Then using interactions from Lagrangian in eq. (2.4) leads to

$$
-i \Sigma_{j i}(p)=\int \frac{d^{4} k}{(2 \pi)^{4}} y_{j \ell} \frac{1}{\left(k^{2}-m_{\ell}^{2}\right)\left((k-p)^{2}-m_{\eta}^{2}\right)} y_{\ell i}=\frac{i}{(4 \pi)^{2}} y_{j \ell} B_{0}\left(p ; m_{\ell}^{2}, m_{\eta}^{2}\right) y_{\ell i},
$$

where the loop function $B_{0}$ (and also $C_{0}, D_{0}, \ldots$ below) is a PV-function defined in section 3.3 .

Next, we consider the corresponding one-loop diagram in flavour basis of eq. (2.1), employing the standard diagrammatic MIA approach. The massive flavour propagators are defined by absorbing the diagonal part of the flavour mass matrix $\mathbf{M}^{2}$, according to the decomposition into diagonal and non-diagonal parts,

$$
M_{I J}^{2}=\left(M_{0}^{2}\right)_{I I} \delta_{I J}+\hat{M}_{I J}^{2}, \quad \hat{M}_{I I}^{2}=0,(\text { no sum over I) },
$$

where $\delta_{I J}$ is the usual Kronecker symmetric tensor. The non-diagonal elements $\hat{M}_{I J}^{2}$, are identified as the mass insertions, treated as perturbative couplings for the non-diagonal quadratic interactions of flavour fields. The one-loop flavour changing self-energy of the flavour states, $\Phi_{I}$, is thus represented as the infinite sum of the diagrams shown in figure 2 . By direct calculation, and denoting $M_{K}^{2} \equiv\left(M_{0}^{2}\right)_{K K}$, one obtains

$$
\begin{aligned}
-i \hat{\Sigma}_{J I}(p)=\int \frac{d^{4} k}{(2 \pi)^{4}} & \frac{Y_{J K} Y_{L I}}{(k-p)^{2}-m_{\eta}^{2}} \times\left(\frac{\delta_{K L}}{k^{2}-M_{K}^{2}}+\frac{\hat{M}_{K L}^{2}}{\left(k^{2}-M_{K}^{2}\right)\left(k^{2}-M_{L}^{2}\right)}\right. \\
& \left.+\frac{\hat{M}_{K N}^{2} \hat{M}_{N L}^{2}}{\left(k^{2}-M_{K}^{2}\right)\left(k^{2}-M_{N}^{2}\right)\left(k^{2}-M_{L}^{2}\right)}+\ldots\right) \\
= & \frac{i}{\left(4 \pi^{2}\right)} Y_{J K} Y_{L I} \times\left(\delta_{K L} B_{0}\left(p ; M_{K}^{2}, m_{\eta}^{2}\right)+\hat{M}_{K L}^{2} C_{0}\left(0, p ; M_{K}^{2}, M_{L}^{2}, m_{\eta}^{2}\right)\right. \\
& \left.+\hat{M}_{K N}^{2} \hat{M}_{N L}^{2} D_{0}\left(0,0, p ; M_{K}^{2}, M_{N}^{2}, M_{L}^{2}, m_{\eta}^{2}\right)+\ldots\right)
\end{aligned}
$$


which is essentially an expansion in terms of mass insertions. We should notice that although $M_{K}^{2}$ are not the squares of the physical masses, they are always real non-negative. This is a general property of the diagonal part of any semi-positive definite Hermitian matrix, including also any Hermitian (squared) mass matrix of a consistent QFT.

As the external indices imply, the self energy diagrams in this example are not invariant under flavour rotations. One can formally uncover the explicit correspondence between the flavour and mass basis calculations by considering the flavour invariance of the time evolution operator, inside the corresponding $S$-scattering matrix element, with the relevant contractions

$$
\begin{aligned}
& \int d^{4} x \int d^{4} y\left\langle p, \overrightarrow{J \mid} \Phi_{J}^{\dagger}(x) \hat{\mathcal{O}}_{(x, y)}^{J I} \Phi_{I} \overleftrightarrow{(y) \mid p^{\prime}}, I\right\rangle=\int d^{4} x \int d^{4} y\left\langle p, \vec{J} \mid \phi_{j}^{\dagger}(x) \mathcal{O}_{(x, y)}^{j i} \phi_{i} \overleftrightarrow{(y) \mid p^{\prime}}, I\right\rangle, \\
& \left\langle p, \vec{J}\left|\phi_{j}^{\dagger}(x)=\left\langle 0\left|e^{i p x} U_{J j}, \phi_{i}(y)\right| p^{\prime}, I\right\rangle=U_{i I}^{\dagger} e^{-i p^{\prime} y}\right| 0\right\rangle,
\end{aligned}
$$

we derive the transformation rule for self-energies,

$$
\hat{\Sigma}_{J I}(p)=U_{J j} \Sigma_{j i}(p) U_{i I}^{\dagger},
$$

which can be immediately generalized to the case of an arbitrary $n$-point amplitude.

Substituting in eq. (2.10) the explicit algebraic expressions for the self-energies we obtain an interesting result - the flavour rotation of the mass eigenstates loop-function is an expansion in terms of mass insertions in flavour basis (no sum over $K, L$ ),

$$
\begin{aligned}
U_{K \ell} B_{0}\left(p, m_{\ell}^{2}, m_{\eta}^{2}\right) U_{\ell L}^{\dagger}= & \delta_{K L} B_{0}\left(p ; M_{K}^{2}, m_{\eta}^{2}\right)+\hat{M}_{K L}^{2} C_{0}\left(0, p ; M_{K}^{2}, M_{L}^{2}, m_{\eta}^{2}\right) \\
& +\hat{M}_{K N}^{2} \hat{M}_{N L}^{2} D_{0}\left(0,0, p ; M_{K}^{2}, M_{N}^{2}, M_{L}^{2}, m_{\eta}^{2}\right)+\ldots
\end{aligned}
$$

This result, however, can be also obtained by a theorem of matrix analysis [cf. eq. (3.11)] stated in the next section, rendering diagrammatic calculations in flavour basis, similar to ones leading to eq. (2.8), obsolete.

\section{Flavour expansion theorem}

Eq. (2.11) has been obtained diagrammatically with the help of the transformation rule (2.10). In what follows we show that, such relations can be also obtained purely algebraically, allowing for an easier transformation between mass and flavour basis calculations without the use of the diagrammatic MIA. In this section we formulate a relevant mathematical framework and a useful general theorem of matrix analysis. For brevity we refer to it as "Flavour Expansion Theorem" or just FET.

Before formulating FET, it is worth noting that obtaining the relation (2.11) in a closed form without reverting to diagrammatic MIA expansion is not easy with the use of standard perturbation techniques. The simplest idea of expanding the mass eigenstates result in a Taylor series around some average mass $m_{0}^{2}=\frac{1}{N} \sum_{K=1}^{N} m_{K}^{2}$,

$$
U_{i K} f\left(m_{K}^{2}\right) U_{j K}^{\star}=U_{i K}\left[\sum_{n=0}^{\infty} \frac{f^{(n)}\left(m_{0}^{2}\right)}{n !}\left(m_{K}^{2}-m_{0}^{2}\right)^{n}\right] U_{j K}^{\star},
$$


fails to reproduce correctly even the first non-trivial MIA term in eq. (2.11) - the higher terms of any order in Taylor expansion are proportional to a factor

$$
U_{i K}\left(m_{K}^{2}-m_{0}^{2}\right)^{n} U_{j K}^{\star}=\left[\left(\mathbf{M}^{\mathbf{2}}-m_{0}^{2} \mathbf{I}\right)^{n}\right]_{i j}=\left[\left(\mathbf{M}_{\mathbf{0}}^{\mathbf{2}}-m_{0}^{2} \mathbf{I}+\hat{\mathbf{M}}^{\mathbf{2}}\right)^{n}\right]_{i j},
$$

which after expanding would explicitly contain terms linear in $\hat{M}_{i j}^{2}$.

Alternatively, a more consistent approach can be developed using the standard quantum mechanic perturbation theory, applied to mass matrix eigenstate problem,

$$
\left(\mathbf{M}_{\mathbf{0}}^{\mathbf{2}}+\lambda \hat{\mathbf{M}}^{\mathbf{2}}\right)\left(\mathbf{e}_{\mathbf{I}}^{(\mathbf{0})}+\lambda \mathbf{e}_{\mathbf{I}}^{(\mathbf{1})}+\ldots\right)=\left[\left(m^{2}\right)_{I}^{(0)}+\lambda\left(m^{2}\right)_{I}^{(1)}+\ldots\right]\left(\mathbf{e}_{\mathbf{I}}^{(\mathbf{0})}+\lambda \lambda \mathbf{e}_{\mathbf{I}}^{(\mathbf{1})}+\ldots\right)
$$

with $\lambda$ being the expansion parameter. By solving eq. (3.3) order by order we find mass eigenvalues $m_{I}^{2}$ and rotation matrices $\mathbf{U}=\left(\mathbf{e}_{\mathbf{1}}, \ldots, \mathbf{e}_{\mathbf{N}}\right)$ as a series in $\lambda$. Then the product, $U_{i K} f\left(m_{K}^{2}\right) U_{f K}^{\star}$, can again be expanded in Taylor series in $\lambda$ parameter, with each term equivalent to the same order of MIA expansion. Such procedure easily restores the first terms in eq. (2.11) (see e.g. [17]), but its combinatorial complication grows quickly and again it is hard to see how the higher order terms combine to form compact $n$-point loop functions, a situation which becomes even trickier in case of degenerate eigenvalues.

\subsection{Hermitian matrix function and divided differences}

We append below definitions that are relevant for presenting the Flavour Expansion Theorem.

Definition 1. Let $\mathbf{A}$ be an $n \times n$ Hermitian matrix, diagonalized by a unitary matrix $\mathbf{U}$ to a real diagonal matrix $\mathbf{D}$, through the transformation,

$$
\mathbf{U}^{\dagger} \mathbf{A} \mathbf{U}=\mathbf{D}=\operatorname{diag}\left(\lambda_{1}, \ldots, \lambda_{n}\right) .
$$

Also let $f(x)$ be a real analytic function about zero, in a range $I \subseteq \mathbb{R}$, that can be expressed in terms of its Maclaurin series, as

$$
f(x)=\sum_{m=0}^{\infty} c_{m} x^{m}
$$

Then, if all $\lambda_{i} \in I$, one can define a Hermitian matrix function $f(\mathbf{A})$, as

$$
\mathbf{U} f(\mathbf{D}) \mathbf{U}^{\dagger}=\sum_{m=0}^{\infty} c_{m} \mathbf{U D}^{m} \mathbf{U}^{\dagger}=\sum c_{m} \mathbf{A}^{m} \equiv f(\mathbf{A}) .
$$

Definition 2. For any function $f(x)$, one can define a set of multi-variable functions, $f^{[k]}$, through a mathematical operation commonly referred to as divided difference. Divided differences are defined recursively as

$$
\begin{aligned}
f^{[0]}(x) & \equiv f(x), \\
f^{[1]}\left(x_{0}, x_{1}\right) & \equiv \frac{f\left(x_{0}\right)-f\left(x_{1}\right)}{x_{0}-x_{1}}, \\
\ldots & \\
f^{[k+1]}\left(x_{0}, \ldots, x_{k}, x_{k+1}\right) & \equiv \frac{f^{[k]}\left(x_{0}, \ldots, x_{k-1}, x_{k}\right)-f^{[k]}\left(x_{0}, \ldots, x_{k-1}, x_{k+1}\right)}{x_{k}-x_{k+1}} .
\end{aligned}
$$


It is easy to check that divided differences of any order $k$ are always totally symmetric under the permutation of any set of respective arguments, $x_{i}$. Moreover, for an analytic generating functional $f=f^{[0]}$ they also have a well defined degeneracy limit

$$
\lim _{\left\{x_{0}, \ldots, x_{m}\right\} \rightarrow\{\xi, \ldots, \xi\}} f^{[k]}\left(x_{0}, \ldots, x_{k}\right)=\frac{1}{m !} \frac{\partial^{m}}{\partial \xi^{m}} f^{[k-m]}\left(\xi, x_{m+1} \ldots, x_{k}\right),
$$

applied to any set of respective arguments $(m \leq k)$, as long as all arguments lie in the domain of analyticity of $f(x)$.

\subsection{The Flavour Expansion Theorem (FET)}

By making extensive use of the definitions above, we can formulate a general theorem, concerning a certain expansion of Hermitian matrix functions, in a form which can be directly applied to the unitary transformation of loop functions in flavour physics.

Theorem 1. Let $\mathbf{A}$ be any $n \times n$ Hermitian matrix, decomposed as a sum of its diagonal and non-diagonal part,

$$
\mathbf{A}=\mathbf{A}_{0}+\hat{\mathbf{A}}
$$

where, by definition,

$$
\begin{aligned}
A_{0}^{I} & \equiv A_{I I}, \\
\hat{A}_{I J} & \equiv A_{I J}, \quad \hat{A}_{I I}=0, \quad(I, J=1, \ldots, n) .
\end{aligned}
$$

Then, for any Hermitian matrix function $f(\mathbf{A})$, satisfying the restrictions of Def. 1, a given matrix element $\langle I|f(A)| J\rangle$ will be given by the expansion (no sum over I,J)

$$
\begin{aligned}
f(\mathbf{A})_{I J}= & \delta_{I J} f\left(A_{0}^{I}\right)+f^{[1]}\left(A_{0}^{I}, A_{0}^{J}\right) \hat{A}_{I J}+\sum_{K_{1}} f^{[2]}\left(A_{0}^{I}, A_{0}^{J}, A_{0}^{K_{1}}\right) \hat{A}_{I K_{1}} \hat{A}_{K_{1} J} \\
& +\sum_{K_{1}, K_{2}} f^{[3]}\left(A_{0}^{I}, A_{0}^{J}, A_{0}^{K_{1}}, A_{0}^{K_{2}}\right) \hat{A}_{I K_{1}} \hat{A}_{K_{1} K_{2}} \hat{A}_{K_{2} J}+\ldots
\end{aligned}
$$

in terms of divided differences of $f\left(\mathbf{A}_{\mathbf{0}}\right)$ [see Def. 2] and the (non-diagonal) elements of $\hat{\mathbf{A}}^{4}$

Eq. 3.11 holds as long as the expansion in the r.h.s. is convergent. The formal proof of this theorem, based on the notion of fully symmetrized polynomials and mathematical induction, is given in appendix A.

\subsection{Divided differences and Passarino-Veltman functions}

The natural connection between FET and the expansion of one-loop amplitudes is becoming striking when looking into the recursive properties of the loop functions in the PassarinoVeltman basis [13]. The general $n$-point one-loop functions can be defined as:

$$
\begin{aligned}
\frac{i}{(4 \pi)^{2}} P V_{n}^{\mu_{1} \ldots \mu_{l}}\left(p_{1}, \ldots, p_{n-1} ; m_{1}^{2}, \ldots, m_{n}^{2}\right)= \\
\quad=\int \frac{d^{4} k}{(2 \pi)^{4}} \frac{k^{\mu_{1}} \ldots k^{\mu_{l}}}{\left(k^{2}-m_{1}^{2}\right) \prod_{j=2}^{n}\left(\left(k+p_{1}+\cdots+p_{j-1}\right)^{2}-m_{j}^{2}\right)}, \quad(n \geq 2) .
\end{aligned}
$$

\footnotetext{
${ }^{4}$ Cases of degenerate eigenvalues and/or diagonal matrix elements are treated uniformly due to property (3.8) of the divided differences.
} 
In the standard notation $n=2,3 \ldots$ functions are commonly denoted as $B, C, \ldots$-functions.

A useful property associates differences of integral functions of a certain order with integral functions of next order. In general case this relation has the following structure:

$$
\begin{gathered}
\frac{P V_{n}^{X}\left(p_{1} \ldots p_{n-1} ; m_{1}^{2} \ldots m_{n}^{2}\right)-P V_{n}^{X}\left(p_{1} \ldots p_{n-1} ; m_{1}^{\prime 2} \ldots m_{n}^{2}\right)}{m_{1}^{2}-m_{1}^{\prime 2}} \\
\quad=P V_{n+1}^{X}\left(0, p_{1} \ldots p_{n-1} ; m_{1}^{2}, m_{1}^{\prime 2} \ldots m_{n}^{2}\right), \quad \\
\frac{P V_{n}^{X}\left(\ldots p_{j-1} \ldots ; \ldots m_{j}^{2} \ldots\right)-P V_{n}^{X}\left(\ldots p_{j-1} \ldots ; \ldots m_{j}^{\prime 2} \ldots\right)}{m_{j}^{2}-m_{j}^{\prime 2}} \\
=P V_{n+1}^{X}\left(\ldots p_{j-1}, 0 \ldots ; \ldots m_{j}^{2}, m_{j}^{\prime 2} \ldots\right), \quad(j \geq 2)
\end{gathered}
$$

with $X$ being any set of Lorentz indices of momenta in the numerator of loop integrand. ${ }^{5}$

Comparing eq. (3.13) with the definition (3.7) one can see immediately that the notion of divided differences is naturally implemented in the relations between multi-point oneloop integrals. Eq. (3.13) allow us to express the expansion (3.11) for one-loop amplitudes in a form in which it is obvious that it is not singular for degenerate diagonal matrix elements. Namely, every one-loop amplitude can be written as a linear combination of PV functions. Furthermore, each PV function can be expanded as,

$$
\begin{aligned}
{\left[P V^{(n)}(\ldots, A, \ldots)\right]_{I J}=} & \delta_{I J} P V^{(n)}\left(\ldots, A_{0}^{I}, \ldots\right)+P V^{(n+1)}\left(\ldots, A_{0}^{I}, A_{0}^{J}, \ldots\right) \hat{A}_{I J} \\
& +\sum_{K} P V^{(n+2)}\left(\ldots, A_{0}^{I}, A_{0}^{J}, A_{0}^{K}, \ldots\right) \hat{A}_{I K} \hat{A}_{K J}+\ldots,
\end{aligned}
$$

where if necessary one should also specify momenta arguments as prescribed in eq. (3.13).

For example, to make a connection between FET and the toy-model of the previous section we observe that if we make the following identifications

$$
\mathbf{D} \rightarrow \mathbf{m}^{\mathbf{2}}, \quad \mathbf{A} \rightarrow \mathbf{M}^{\mathbf{2}}, \quad f(x) \equiv f^{[0]}(x) \rightarrow B_{0}\left(p, x, m_{\eta}^{2}\right),
$$

we can immediately see that eq. (2.11) is a special case of eq. (3.11). In section 5 , we will present a highly non-trivial example application of the FET.

\subsection{Applications and limitations of FET}

The FET formulated as a pure algebraic theorem can be directly applied to expanding a mass eigenstate result of any transition amplitude in any model involving particles associated with Hermitian mass matrices, that is scalars or vectors, even at higher loop orders. As we shall discuss in section 4, with some modifications, FET can be also applied to expanding the amplitudes involving fermions associated with non-Hermitian mass matrices.

The purely algebraic expansion is usually significantly simpler than the more tedious and prone to mistakes diagrammatic MIA calculation, particularly in models with complicated flavour structure like MSSM. Another advantage of FET is that it can be easily

\footnotetext{
${ }^{5}$ Additional discussion and more recursive relations for the various types of PV functions can be found in appendix A of ref. [18].
} 
implemented as an algorithm for symbolic manipulation programs, automatizing the expansion procedure. However, the procedure has some limitations, particularly when is applied to such complicated functions as loop integrals. Three remarks concerning limitations of FET are summarized here:

Remark 1. FET assumptions require the expanded amplitude to be analytic function of masses. This is not the case if external momenta are large and loop integrals may have branch cuts. For the external momenta in the vicinity of branch cuts a mass eigenstate calculation and use of numerical procedures is more appropriate.

Remark 2. Flavour expansion in the r.h.s. of eq. (3.11) may not converge or converge very slowly, in both cases again mass eigenstates basis and use of numerical procedures is preferred.

Remark 3. The UV-singularities do not pose a problem for FET. If they appear, they come from the loop integrals of positive mass dimension. The coefficients of poles of such integrals are dimensionless and flavour blind or proportional to positive powers of masses, so they can be evaluated in terms of flavour basis parameters without any expansion.

Most practical applications of FET concern analyses of models of New Physics where loop particles are much heavier than the external states (being usually the Standard Model fields). Thus, it is usually sufficient to calculate relevant amplitudes in the approximation of vanishing external momenta, or by expanding the loop integrals in the external momenta and keeping only the first few terms of such an expansion. Since in these processes the loop integrals are real analytic functions of masses, branch cuts can never appear. Then, the only remaining problem is the convergence of the FET.

In appendix B, we formulate and prove the condition which has to be fulfilled by the mass matrices in the flavour basis, in order to make FET written for any one-loop amplitude, convergent. The result is that the moduli of every eigenvalue of the dimensionless mass insertion matrix has to be smaller than one.

\section{Expansion of fermionic amplitudes}

Expanding amplitudes in which flavour violation enters through fermionic mass matrices is more complicated, because such matrices do not need to be Hermitian and in general can be diagonalized with the use of two different unitary matrices. Nevertheless, as it turns out, FET can always apply to this case as well, with minor, but necessary, modifications which we discuss below.

Lets first consider a Lagrangian of $N$-copies of Dirac fermion free fields. This will have the general form,

$$
\begin{aligned}
\mathcal{L}_{\text {flavour }}^{(0)} & =i \bar{\Psi}_{A} \not \partial \Psi_{A}-M_{A B}\left(\bar{\Psi}_{A} P_{L} \Psi_{B}\right)-M_{A B}^{\dagger}\left(\bar{\Psi}_{A} P_{R} \Psi_{B}\right) \\
& \equiv \bar{\Psi}\left(i \not \partial-\mathbf{M} P_{L}-\mathbf{M}^{\dagger} P_{R}\right) \Psi
\end{aligned}
$$

in a 4-spinor Dirac notation, which is more suitable for mass eigenstates calculations and offers a more compact description in our following discussion. Since Majorana spinors can 
be understood as Dirac spinors with an extra chirality constraint, our discussion applies directly to Majorana fermions, as well.

As is well known, in a chiral theory, a Dirac spinor is in general reducible under flavour rotations. The transformation from flavour to mass basis is performed through two different unitary matrices, acting independently on its chiral projections, as

$$
\Psi_{L A}=U_{A i} \psi_{L i}, \quad \Psi_{R A}=V_{A i} \psi_{R i},
$$

which can always bring an arbitrary complex flavour mass matrix $\mathbf{M}$ into a real nonnegative diagonal form, satisfying

$$
\mathbf{V}^{\dagger} \mathbf{M} \mathbf{U}=\mathbf{m}=\operatorname{diag}\left(m_{1}, \ldots, m_{N}\right) .
$$

The unitary matrices $\mathbf{V}$ and $\mathbf{U}$ diagonalize also the (semi) positive-definite Hermitian matrices $\mathbf{M} \mathbf{M}^{\dagger}$ and $\mathbf{M}^{\dagger} \mathbf{M}$, through the transformations

$$
\mathbf{V}^{\dagger} \mathbf{M} \mathbf{M}^{\dagger} \mathbf{V}=\mathbf{U}^{\dagger} \mathbf{M}^{\dagger} \mathbf{M} \mathbf{U}=\mathbf{m}^{2}
$$

To streamline the notation, we introduce the unitary matrices $\mathcal{U}$ and $\overline{\mathcal{U}}$, generalizing our transformation rules for chirality projected fermion fields to a reducible Dirac 4 -spinor, as

$$
\mathcal{U} \equiv \mathbf{U} P_{L}+\mathbf{V} P_{R}, \quad \overline{\mathcal{U}} \equiv \mathbf{U}^{\dagger} P_{R}+\mathbf{V}^{\dagger} P_{L}
$$

In this compact description, eq. (4.3) will result in

$$
\Psi_{A}=\mathcal{U}_{A i} \psi_{i}, \quad \bar{\Psi}_{A}=\bar{\psi}_{i} \overline{\mathcal{U}}_{i A} .
$$

The free propagator for the fermion multiplet $\boldsymbol{\Psi}$ in flavour basis is a matrix both in spinor and flavour space. Inverting the Dirac operator in eq. (4.2), we find

$$
\begin{aligned}
\hat{\boldsymbol{\Delta}}(k) & =\frac{i}{\not k-\mathbf{M} P_{L}-\mathbf{M}^{\dagger} P_{R}} \\
& =\left(\mathbf{M}^{\dagger} P_{L}+\not k P_{L}\right) \frac{i}{k^{2}-\mathbf{M M}^{\dagger}}+\left(\mathbf{M} P_{R}+\not k P_{R}\right) \frac{i}{k^{2}-\mathbf{M}^{\dagger} \mathbf{M}} .
\end{aligned}
$$

The free propagators in flavour and mass eigenstates basis are related by the same rotations as fermion fields. From the identity,

$$
\left\langle 0\left|T\left\{\Psi_{B}(x) \bar{\Psi}_{A}(y)\right\}\right| 0\right\rangle=\mathcal{U}_{B i}\left\langle 0\left|T\left\{\psi_{i}(x) \bar{\psi}_{i}(y)\right\}\right| 0\right\rangle \overline{\mathcal{U}}_{i A},
$$

it follows that,

$$
(\hat{\boldsymbol{\Delta}}(k))_{B A}=(\mathcal{U} \boldsymbol{\Delta}(k) \overline{\mathcal{U}})_{B A}=\left(\mathcal{U} \frac{i(\not k+\mathbf{m})}{k^{2}-\mathbf{m}^{\mathbf{2}}} \overline{\mathcal{U}}\right)_{B A},
$$

where $\Delta_{i}(k)$ is the fermion propagator in the mass eigenstates basis. Applying the explicit expressions of eq. (4.6) for the reducible flavour rotation matrices, to eq. (4.10) and using the following algebraic identities:

$$
\begin{aligned}
\overline{\mathcal{U}}^{\dagger} \frac{1}{k^{2}-\mathbf{m}^{\mathbf{2}}} \overline{\mathcal{U}} & =\frac{1}{k^{2}-\mathbf{M} \mathbf{M}^{\dagger}} P_{L}+\frac{1}{k^{2}-\mathbf{M}^{\dagger} \mathbf{M}} P_{R}, \\
\mathcal{U} \frac{\mathbf{m}}{k^{2}-\mathbf{m}^{\mathbf{2}}} \overline{\mathcal{U}} & =\mathbf{M}^{\dagger} \frac{1}{k^{2}-\mathbf{M M}^{\dagger}} P_{L}+\mathbf{M} \frac{1}{k^{2}-\mathbf{M}^{\dagger} \mathbf{M}} P_{R},
\end{aligned}
$$


the flavour propagator $\hat{\boldsymbol{\Delta}}(k)$ can be also obtained from the mass basis propagator $\boldsymbol{\Delta}(k)$.

In order to calculate the amplitude, apart from propagators one needs to consider the transformation rules for the fermionic vertices. A general fermionic current in flavour basis, can be expressed in the form,

$$
j_{\Psi}=\bar{\Psi}_{A} \hat{\Gamma}_{A B} \Psi_{B},
$$

where $\hat{\boldsymbol{\Gamma}}$ is an operator acting both in flavour and spinor space, and may also depend on scalar or gauge fields. In any QFT model a general fermionic vertex can be decomposed into four chirality projected terms as

$$
\begin{aligned}
\hat{\boldsymbol{\Gamma}} & =\hat{\boldsymbol{\Gamma}}_{R L} P_{L}+\hat{\boldsymbol{\Gamma}}_{L R} P_{R}+\hat{\boldsymbol{\Gamma}}_{R R} P_{R}+\hat{\boldsymbol{\Gamma}}_{L L} P_{L} \\
& \equiv P_{L} \hat{\boldsymbol{\Gamma}}_{R L}+P_{R} \hat{\boldsymbol{\Gamma}}_{L R}+P_{L} \hat{\boldsymbol{\Gamma}}_{R R}+P_{R} \hat{\boldsymbol{\Gamma}}_{L L} .
\end{aligned}
$$

where $\hat{\boldsymbol{\Gamma}}_{L R(R L)}$ are scalar- or tensor-type couplings, and $\hat{\boldsymbol{\Gamma}}_{L L(R R)}$ are vector couplings.

In our compact notation, the transformation rule for vertices can be simply expressed as:

$$
\hat{\Gamma}=\overline{\mathcal{U}}^{\dagger} \boldsymbol{\Gamma} \mathcal{U}^{\dagger}
$$

or explicitly in terms of $\hat{\boldsymbol{\Gamma}}$ components as

$$
\begin{array}{ll}
\hat{\boldsymbol{\Gamma}}_{R L}=\mathbf{V} \boldsymbol{\Gamma}_{R L} \mathbf{U}^{\dagger}, & \hat{\boldsymbol{\Gamma}}_{R R}=\mathbf{V} \boldsymbol{\Gamma}_{R R} \mathbf{V}^{\dagger} \\
\hat{\boldsymbol{\Gamma}}_{L R}=\mathbf{U} \boldsymbol{\Gamma}_{L R} \mathbf{V}^{\dagger}, & \hat{\boldsymbol{\Gamma}}_{L L}=\mathbf{U} \boldsymbol{\Gamma}_{L L} \mathbf{U}^{\dagger}
\end{array}
$$

It is important to notice that the transformation rules for propagators and for vertices eqs. (4.10) and (4.15), respectively, are different, which reflects the general difference in transformation rules for amputated and non-amputated Green's functions.

Let us examine now the general $n$-point transition amplitude with fermion lines (external or internal - our formalism applies to the latter by setting final and initial fermion indices to be equal). Lets focus on any chosen fermion line in such an amplitude. The Feynman rule in the mass eigenstate basis would have the general form,

$$
\mathcal{M}_{j i} \sim(\Gamma \Delta \Gamma \ldots \Delta \Gamma)_{j i}
$$

Applying flavour rotation to the external states and using eqs. (4.10) and (4.15)), one can get an expression for the fermion line in flavour basis, $\hat{\mathcal{M}}_{J I}$, written as a sequential product of flavour-basis fermion vertices and matrix propagators,

$$
\begin{aligned}
\hat{\mathcal{M}} & =\overline{\mathcal{U}}^{\dagger} \mathcal{M} \mathcal{U}^{\dagger} \sim\left(\overline{\mathcal{U}}^{\dagger} \boldsymbol{\Gamma} \mathcal{U}^{\dagger}\right)(\mathcal{U} \Delta \overline{\mathcal{U}})\left(\overline{\mathcal{U}}^{\dagger} \boldsymbol{\Gamma} \mathcal{U}^{\dagger}\right) \ldots(\mathcal{U} \Delta \overline{\mathcal{U}})\left(\overline{\mathcal{U}}^{\dagger} \boldsymbol{\Gamma} \mathcal{U}^{\dagger}\right) \\
& =\hat{\Gamma} \hat{\Delta} \hat{\Gamma} \ldots \hat{\boldsymbol{\Delta}} \hat{\Gamma} .
\end{aligned}
$$

This shows that any fermionic amplitude built of vertices and propagators in the mass basis can be formally transformed into the amplitude given in terms of respective quantities in the flavour basis. We can now observe that, as shown explicitly in eq. (4.8), matrix denominators of the loop integrals in the flavour basis always depend on Hermitian 
matrices $\mathbf{M} \mathbf{M}^{\dagger}$ or $\mathbf{M}^{\dagger} \mathbf{M}$, and only such combinations would appear as formal arguments of loop functions. As a consequence, one can conclude that FET formulated for Hermitian matrices can always apply to loop functions appearing in fermionic amplitudes, as well.

From more practical point of view, our derivation leads to the conclusion that the fermion mixing matrices $\mathbf{U}$ and $\mathbf{V}$ can appear in amplitudes only is some specific combinations, namely

$$
\begin{aligned}
U_{B i} f\left(m_{i}^{2}\right) U_{A i}^{\star} & =f\left(\mathbf{M}^{\dagger} \mathbf{M}\right)_{B A}, \\
V_{B i} f\left(m_{i}^{2}\right) V_{A i}^{\star} & =f\left(\mathbf{M} \mathbf{M}^{\dagger}\right)_{B A}, \\
U_{B i} m_{i} f\left(m_{i}^{2}\right) V_{A i}^{\star} & =M_{B C}^{\dagger} f\left(\mathbf{M} \mathbf{M}^{\dagger}\right)_{C A}=f\left(\mathbf{M}^{\dagger} \mathbf{M}\right)_{B C} M_{C A}^{\dagger}, \\
V_{B i} m_{i} f\left(m_{i}^{2}\right) U_{A i}^{\star} & =M_{B C} f\left(\mathbf{M}^{\dagger} \mathbf{M}\right)_{C A}=f\left(\mathbf{M M}^{\dagger}\right)_{B C} M_{C A},
\end{aligned}
$$

which can always be expanded using eq. (3.11).

We should notice that the formal treatment followed in this section can easily generalize to the case of more complicated flavour models, where sets of flavour fields belong to distinct flavour families. In this case, the propagators and the vertices in the general formulae of eqs. (4.17) and (4.18), will carry both internal (flavour) and external (family-group) indices. However, only $\boldsymbol{\Gamma}$ or $\hat{\boldsymbol{\Gamma}}$ can associate different family groups because propagators, $\boldsymbol{\Delta}(k)$ or $\hat{\boldsymbol{\Delta}}(k)$ are block diagonal in family space. Therefore, one can accommodate in this formalism amplitudes with complicated flavour structure like, e.g.,rare processes in the MSSM with fermions on the external lines and sfermions and gauginos circulating in loops. FET formalism not only allows one to calculate such diagrams in flavour basis but also in any other "hybrid" basis of convenience, e.g.,fermions-gauginos in mass and sfermions in flavour basis, or any other combination.

\section{Application of FET: neutron EDM in the MSSM}

To illustrate that higher order mass insertion terms can give physically meaningful bounds we consider the example of the neutron Electric Dipole Moment (nEDM) in the Minimal Supersymmetric Standard Model [19-21].

The full $\mathrm{nEDM}$ can be expressed as a combination of parton level contributions - EDMs of quarks $d_{q}$, their chromoelectric dipole moments (CDM) $c_{q}$ and the CDM of gluon $c_{g}$. The parton moments are defined as respective coefficients in the effective Hamiltonian:

$$
\begin{aligned}
& \mathcal{H}_{q}=\frac{i d_{q}}{2} \bar{q} \sigma_{\mu \nu} \gamma_{5} q F^{\mu \nu}-\frac{i c_{q}}{2} \bar{q} \sigma_{\mu \nu} \gamma_{5} T^{a} q G^{\mu \nu a}, \\
& \mathcal{H}_{g}=-\frac{c_{g}}{6} f_{a b c} G_{\mu \rho}^{a} G_{\nu}^{b \rho} G_{\lambda \sigma}^{c} \epsilon^{\mu \nu \lambda \sigma} .
\end{aligned}
$$

The total neutron EDM depend on its hadronic wave function and can be written as

$$
E_{n}=\eta_{e d} d_{d}+\eta_{e u} d_{u}+e\left(\eta_{c d} c_{d}+\eta_{c u} c_{u}\right)+\frac{e \eta_{g} \Lambda_{X}}{4 \pi} c_{g}
$$

where $\eta_{i}$ and $\Lambda_{X}$ are $\mathcal{O}(1)$ QCD wave-function factors [22] and the chiral symmetry breaking scale [23], respectively. Various models can give significantly different values for $\eta_{i}$, differing 
even by an overall sign. Thus, eq. (5.2) and the bounds it puts on MSSM parameters should be treated as order of magnitude estimates only, since potential cancellations in (5.2) depend on these poorly known coefficients.

The explicit expressions for $d_{q}, c_{q}$ and $c_{g}$ are given in ref. [24]. In this example, we consider only the dominant gluino contribution to the up-quark EDM and CDM. Taken together, their contribution to the nEDM can be expressed as

$$
E_{n}=\frac{1}{M_{3}} \sum_{k=1}^{6} \operatorname{Im}\left(Z_{U}^{1 k} Z_{U}^{4 k \star}\right) F\left(x_{\tilde{U}_{k}}\right),
$$

where $Z_{U}$ and $m_{\tilde{U}_{k}}$ are up-squark mixing matrices and physical masses, $M_{3}$ is the gluino mass (for conventions and the detailed definitions see refs. [25, 26]), and we define the mass ratios, $x_{\tilde{Q}} \equiv m_{\tilde{Q}}^{2} / M_{3}^{2}$. The function $F(x)$ is the sum of loop contributions

$$
F(x)=\frac{e \alpha_{s}}{18 \pi}\left(8 \eta_{e u} C_{12}(x)-\frac{3 g_{s} \eta_{c u}}{2}\left(18 C_{11}(x)+C_{12}(x)\right)\right),
$$

with $C_{11}, C_{12}$ being certain PV-functions defined as

$$
\begin{aligned}
& C_{11}(x)=\frac{-1+3 x}{4(1-x)^{2}}+\frac{x^{2}}{2(1-x)^{3}} \log x, \\
& C_{12}(x)=-\frac{x+1}{2(1-x)^{2}}-\frac{x}{(1-x)^{3}} \log x .
\end{aligned}
$$

Flavour violation in the MSSM squark sector is strongly bounded by numerous experiments and known to be very small, $\lesssim O\left(10^{-3}\right)$, for down squark mass matrices if the diagonal elements of those matrices are around the electroweak scale. Therefore, we assume for the purposes of this example that the left down soft SUSY breaking squark mass matrix is approximately diagonal, but not degenerate, of the form

$$
\left(m_{\tilde{D}}^{2}\right)_{L L}=\left(\begin{array}{ccc}
m_{\tilde{D}}^{2} & 0 & 0 \\
0 & m_{\tilde{D}}^{2}+\delta m_{D 12}^{2} & 0 \\
0 & 0 & m_{\tilde{D}}^{2}+\delta m_{D 13}^{2}
\end{array}\right) .
$$

In the left up-squark sector the off-diagonal mass terms are then generated by the $\mathrm{SU}(2)$ relation:

$$
\left(m_{\tilde{U}}^{2}\right)_{L L}=K\left(m_{\tilde{D}}^{2}\right)_{L L} K^{\dagger}
$$

where $K$ denotes the Cabibbo-Kobayashi-Maskawa (CKM) matrix.

Consider now the flavour expansion of eq. (5.3). In the first order it constrains the imaginary part of the trilinear up-squark mixing,

$$
E_{n}^{(1)} \supset-\frac{v_{2}}{M_{3}^{3} \sqrt{2}} \operatorname{Im}\left(A_{U}^{11}+Y_{u} \mu^{*} \cot \beta\right) F^{[1]}\left(x_{\tilde{U}_{L 1}}, x_{\tilde{U}_{R 1}}\right),
$$

where the r.h.s. is now expressed in terms of parameters in flavour basis. In particular, the arguments of the first divided difference, $F^{[1]}$, are given by diagonal elements of up-squark mass matrix,

$$
x_{\tilde{U}_{L}} \equiv \frac{\left(m_{\tilde{U}}^{2}\right)_{L L}^{11}}{M_{3}^{2}}, x_{\tilde{U}_{R}} \equiv \frac{\left(m_{\tilde{U}}^{2}\right)_{R R}^{11}}{M_{3}^{2}} .
$$


Following the current experimental bound, $\left|E_{n}\right|<2.9 \times 10^{-26}$ [27], and bearing in mind potential cancellations, eq. (5.9) sets strong bounds on the imaginary phases of $\mu$ and $A_{U}^{11}$, of the order of $10^{-3}$ and $10^{-5}$, respectively, for SUSY mass scale of the order of $1 \mathrm{TeV}$.

What is interesting, and to our knowledge has not been discussed thus far in the literature, is that the experimental bound on $\mathrm{nEDM}$ is so strong that it constrains significantly also the real parts of up-squark mass insertions, an effect which is easily visible when analyzing higher orders in MIA expansion. To avoid lengthy expressions, let us assume that in the up-squark sector only the 31 off-diagonal entries do not vanish in the "right-handed" soft mass matrix $\left(m_{\tilde{U}}^{2}\right)_{R R}^{31}$ and in the trilinear mixing matrix $A_{u}^{31}$ and that they are purely real. In addition, the $\left(m_{\tilde{U}}^{2}\right)_{L L}$ is defined by the relation to diagonal down sector in eq. (5.8). Then, a non-vanishing contributions to nEDM are generated from higher orders in mass insertions via the mixing with the complex CKM matrix elements. Using the FET up to 2nd order one can see that the result depends only on the $A_{u}^{31}$,

$$
\begin{aligned}
E_{n}^{(2)} \supset \frac{v_{2} \sin 2 \theta_{13} \cos \theta_{23}}{2 \sqrt{2} M_{3}^{5}}\left(\delta m_{D 13}^{2}-\right. & \left.\delta m_{D 12}^{2} \sin ^{2} \theta_{12}\right) \\
& \times \sin \delta_{\mathrm{CKM}} \operatorname{Re} A_{u}^{31} F^{[2]}\left(x_{\tilde{U}_{L 1}}, x_{\tilde{U}_{R 1}}, x_{\tilde{U}_{L 3}}\right),
\end{aligned}
$$

where $\theta_{12}, \theta_{13}, \theta_{23}$, and $\delta_{\mathrm{CKM}}$ are respectively, the angles and the CP-violating phase in the standard CKM matrix parametrization. Note again that a CP-violating observable constraints real squark mass parameters through the CKM CP-violating phase.

It is worth noting that even the 3rd order expansion of FET sets numerically significant constraints on the real parts of flavour violating parameters. In particular, the dependence on $\left(m_{\tilde{U}}^{2}\right)_{R R}^{31}$ parameter, absent at lower orders, is now introduced through,

$$
\begin{aligned}
E_{n}^{(3)} \supset \frac{v_{2} \sin 2 \theta_{13} \cos \theta_{23}}{2 \sqrt{2} M_{3}^{7}}\left(\delta m_{D 13}^{2}-\delta m_{D 12}^{2} \sin ^{2} \theta_{12}\right) \sin \delta_{\mathrm{CKM}} \times \\
\quad \times \operatorname{Re}\left(m_{\tilde{U}}^{2}\right)_{R R}^{31} \operatorname{Re}\left(A_{U}^{33}+Y_{t} \mu^{*} \cot \beta\right) F^{[3]}\left(x_{\tilde{U}_{L 1}}, x_{\tilde{U}_{R 1}}, x_{\tilde{U}_{L 3}}, x_{\tilde{U}_{R 3}}\right) .
\end{aligned}
$$

Comparing separately expressions given in eqs. (5.11) and (5.12) with the experimental upper bound on the neutron EDM, one can obtain order of magnitude estimates on, otherwise weakly constrained, 31 and 13 elements of the up-squark trilinear and "right-handed" soft mass terms in relation to mass splitting in the down-squark sector. Such bounds are important e.g. for analysis of the maximal allowed decay rates of the top quark to lighter MSSM Higgs boson, $t \rightarrow u h$ [18]. The numerical results for a typical MSSM parameter set, obtained using the full unexpanded mass eigenstates expressions for $\mathrm{nEDM}$ and the SUSY_FLAVOR library [28-32], are collected in table 1. They all agree both qualitatively and quantitatively with eqs. (5.11) and (5.12) that have been obtained from the FET of eq. (3.11).

Alternatively, one can satisfy the nEDM bound by choosing flavour violating entries large but correlated so that terms in eqs. (5.11) and (5.12) cancel each other to large accuracy. It is interesting to observe that CKM-related factors in these equations are identical, so the correlation between $A_{U}^{31}$ and $\left(m_{\tilde{U}}^{2}\right)_{R R}^{31}$ is given only by SUSY parameters 


\begin{tabular}{|r|c|c|c|c|c|}
\hline$\delta m_{D 13}[\mathrm{TeV}]$ & 0.2 & 0.4 & 0.6 & 0.8 & 1 \\
\hline$\left|\operatorname{Re} A_{U}^{31} / M_{3}\right|$ & $2.4 \times 10^{-2}$ & $1.3 \times 10^{-2}$ & $1.1 \times 10^{-2}$ & $8.9 \times 10^{-3}$ & $7.9 \times 10^{-3}$ \\
$\left|\operatorname{Re}\left(m_{\tilde{U}}^{2}\right)_{R R}^{31} / M_{3}^{2}\right|$ & $2.5 \times 10^{-2}$ & $1.2 \times 10^{-2}$ & $1.1 \times 10^{-2}$ & $8.6 \times 10^{-3}$ & $7.4 \times 10^{-3}$ \\
\hline
\end{tabular}

Table 1. Upper bounds on $\left|\operatorname{Re} A_{U}^{31} / M_{3}\right|$ and $\left|\operatorname{Re}\left(m_{\tilde{U}}^{2}\right)_{R R}^{31} / M_{3}^{2}\right|$ imposed by current experimental constraints from neutron EDM. Displayed values were obtained assuming $\left(m_{\tilde{U}}^{2}\right)_{R R}^{31}=0$ for the 2nd row, $A_{U}^{31}=0$ for the 3rd row and no other sources of the sfermion flavour violation. Other parameters set to $\tan \beta=4$, common SUSY-scale $M_{3}=1.1 \mathrm{TeV}$ and a suitable value of $A_{U}^{33}$ was implicitly chosen to satisfy the $125 \mathrm{GeV}$ Higgs mass constraint.

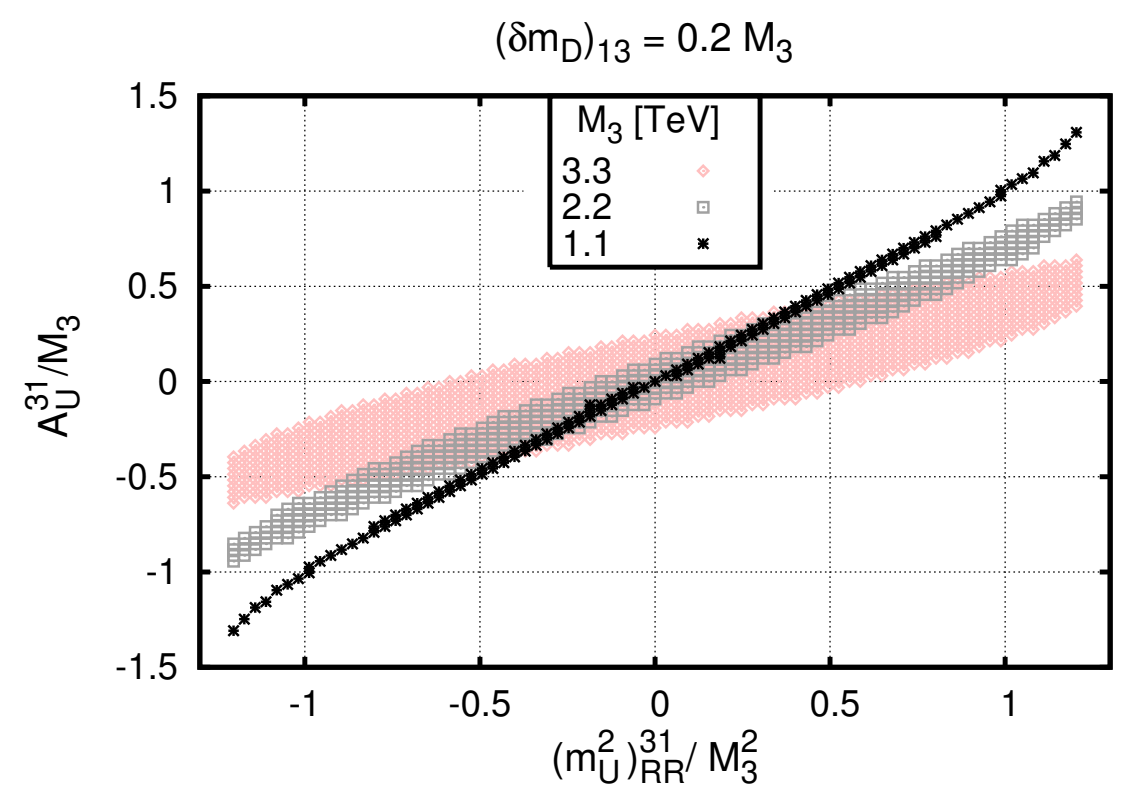

Figure 3. Range of the $\operatorname{Re} A_{U}^{31} / M_{3}-\operatorname{Re}\left(m_{\tilde{U}}^{2}\right)_{R R}^{31} / M_{3}^{2}$ plane allowed by current experimental constraints from neutron EDM. MSSM parameters defined as in table 1.

(of course once the QCD-related factors in eq. (5.4) become better known). Both terms exactly cancel when

$$
\frac{\operatorname{Re}\left[A_{u}^{31} / M_{3}\right]}{\operatorname{Re}\left[\left(m_{\tilde{U}}^{2}\right)_{R R}^{13} / M_{3}^{2}\right]}=-\frac{A_{U}^{33}+Y_{t} \mu^{*} \cot \beta}{M_{3}} \frac{F^{[3]}\left(x_{\tilde{U}_{L 1}}, x_{\tilde{U}_{R 1}}, x_{\tilde{U}_{L 3}}, x_{\tilde{U}_{R 3}}\right)}{F^{[2]}\left(x_{\tilde{U}_{L 1}}, x_{\tilde{U}_{R 1}}, x_{\tilde{U}_{L 3}}\right)} .
$$

Eq. (5.13) suggests a linear shape for the allowed $\operatorname{Re} A_{U}^{31}-\operatorname{Re}\left(m_{\tilde{U}}^{2}\right)_{R R}^{31}$ parameter space, consistent with the nEDM experimental bound. This result is illustrated clearly in figure 3, obtained again with the SUSY_FLAVOR code (i.e.,without using the MIA expansion), and assuming values of QCD factors implemented there. Again this result follows closely eqs. (5.11) and (5.12) that have been obtained from the FET. In particular, numerical comparison between approximate formulae eqs. (5.11) and (5.12) and the exact mass eigenstates calculation implemented in SUSY_FLAVOR, exhibit a remarkable agreement, above $98 \%$, in the central region of figure 3 , namely for $\left(\operatorname{Re} A_{U}^{31} / M_{3}\right) \sim\left(\operatorname{Re}\left(m_{\tilde{U}}^{2}\right)_{R R}^{31} / M_{3}^{2}\right) \sim 5 \times 10^{-2}$, while 
even for very large values of the flavor violating parameters, $(\sim 0.5)$, agreement remains above $85 \%$.

Furthermore, we have successfully applied FET to another observable, namely the rare top decay to light quarks and a Higgs boson, $t \rightarrow q h$, in MSSM [18]. What one practically gains from using FET in complicated theories, like MSSM for example, is to algebraically isolate the dominant effects for a given observable without performing tedious diagrammatic MIA expansion nor extensive computer scans of a multi-parameters space.

\section{Summary}

In this article we have presented and proved a theorem of matrix analysis, the Flavour Expansion Theorem (FET), that remarkably translates any transition amplitude, obtained in terms of mass eigenstate basis parameters, into its corresponding amplitude in flavour eigenstate basis, purely algebraically, without the use of standard diagrammatic methods like the Mass Insertion Approximation (MIA) method. Following the formulation of this theorem, any analytic function of a Hermitian matrix is expanded in terms of its offdiagonal elements with coefficients being the multi-variable functions, commonly known as divided differences. Natural implementation of such expansion [see eq. (3.11)] comes from the intimate connection between the divided differences and the Passarino-Veltman oneloop functions. Apart from the formal proof, we have discussed also FET limitations, such as non-analyticity and convergence issues. We have also extended the use of the theorem in case of general transition amplitudes involving fermion mass matrices which are not necessarily Hermitian.

We have argued many times throughout this article, that the algebraic derivation of the flavour basis result using FET is substantially easier, shorter and more compact than the diagrammatic one. A pedagogical example is given in section 2. However, we also illustrate how FET works with a significant example based on sparticle (gluon-squark) contributions to neutron-EDMs. In this example, the use of FET at higher non-trivial orders is capable to set fairly strong bounds of order $\sim 10^{-2}$ on real parts of up-squark mixing matrix elements from $\mathrm{nEDM}$ measurements by assuming that $\mathrm{CP}$-violation arises only from the CKM-matrix phase. This FET result agrees with our exact numerical calculations [see table 1 and figure 3 ] using SUSY_FLAVOR library. To our knowledge these bounds are new in the MSSM flavour physics literature and demonstrate the usefulness of the Flavour Expansion Theorem, especially, when it applies to complicated models.

\section{Acknowledgments}

J. R. would like to thank University of Ioannina for the hospitality during his stay there. His work was supported in part by the Polish National Science Center under the research grant DEC-2012/05/B/ST2/02597. A. D. would like to thank A. Romanino for useful communication. M. P. would like to thank Klaus Bering for valuable discussions. This research has been co-financed by the European Union (European Social Fund - ESF) and Greek national funds through the Operational Program "Education and Lifelong Learning" 
of the National Strategic Reference Framework (NSRF) - Research Funding Programs: THALIS and ARISTEIA - Investing in the society of knowledge through the European Social Fund.

\section{A Proof of the Flavour Expansion Theorem}

The FET theorem formulated in section 3.2 can be proved using mathematical induction and the notion of the "fully symmetrized polynomials".

\section{A.1 Fully symmetrized polynomials}

The "fully symmetrized" homogeneous polynomials of degree $N$ [33], can be understood through the following equivalent definitions:

Definition 3. $Q_{N}^{M}\left(x_{1}, \ldots, x_{M}\right)$ is the direct sum of all distinct $N$-degree monomials constructed out of the given set of $M$ variables $x_{i}$.

Definition 4. Alternatively, $Q_{N}^{M}\left(x_{1}, \ldots, x_{M}\right)$ is defined as

$$
\left.Q_{N}^{M}\left(x_{1}, \ldots, x_{M}\right) \equiv \sum_{N} x_{1}^{a_{1}} \ldots x_{M}^{a_{M}} \equiv \sum_{a_{1}, \ldots, a_{M}=0}^{N}\left(x_{1}^{a_{1}} \ldots x_{M}^{a_{M}}\right)\right|_{a_{1}+\ldots+a_{M}=N}
$$

Directly from definitions above, one can express the fully symmetrized polynomial, $Q_{N}^{M}$, for any value of $M, N$. Due to the symmetric nature of eq. (A.1) there exist many equivalent representations of this sum. For non trivial $M, N$, one of these will have the explicit form

$$
\begin{aligned}
& Q_{N}^{M}\left(x_{1}, \ldots, x_{M}\right)= \\
& \left(x_{1}\right)^{N}+\left(x_{1}\right)^{N-1}\left(x_{2}+x_{3}+\ldots\right)+\left(x_{1}\right)^{N-2}\left(x_{2}^{2}+\ldots+\left(x_{2} x_{3}\right)+\ldots\right)+\ldots+x_{1}(\ldots) \\
& +\left(x_{2}\right)^{N}+\left(x_{2}\right)^{N-1}\left(x_{3}+\ldots\right)+\ldots \\
& +\left(x_{M}\right)^{N} .
\end{aligned}
$$

The identities, $Q_{N}^{0}=0, Q_{0}^{M}=1, Q_{N}^{1}\left(x_{1}\right)=x_{1}^{N}$, also hold trivially by definition.

Due to eq. (A.1) the factorization property,

$$
Q_{N}^{M}\left(x_{1}, \ldots, x_{M}\right)=\sum_{K=0}^{N} Q_{K}^{L}\left(x_{1}, \ldots, x_{L}\right) Q_{N-K}^{M-L}\left(x_{L+1}, \ldots, x_{M}\right), \quad(\text { no sum over } L),
$$

holds for any integer $L$, satisfying $1 \leq L \leq M-1$, and for any choice of, $\{L\}$ and $\{M-L\}$, respective subsets of $M$ variables, $x_{i}$. 
Lemma 1. Fully symmetrized polynomials $Q_{N}^{M+1}$ are $M$-order divided differences of the generating functional, $Q_{N+M}^{1}$, thus satisfying $Q_{N}^{M+1}=Q_{N+M}^{1[M]}$. Equivalently, the expression,

$$
Q_{N}^{M+1}=Q_{N+1}^{M[1]}
$$

holds for any $M \geq 1$.

Proof. First, we show the validity of eq. (A.4), for $M=1$, namely

$$
Q_{N}^{2}=Q_{N+1}^{1[1]} .
$$

Applying eq. (A.3), we have

$$
\begin{aligned}
\left(x_{1}-x_{2}\right) Q_{N}^{2}\left(x_{1}, x_{2}\right)= & \sum_{K=0}^{N}\left(Q_{N-K+1}^{1}\left(x_{1}\right) Q_{K}^{1}\left(x_{2}\right)-Q_{K}^{1}\left(x_{1}\right) Q_{N-K+1}^{1}\left(x_{2}\right)\right) \\
= & Q_{N+1}^{1}\left(x_{1}\right)-Q_{N+1}^{1}\left(x_{2}\right)+\sum_{K=1}^{N} Q_{N-K+1}^{1}\left(x_{1}\right) Q_{K}^{1}\left(x_{2}\right) \\
& \quad-\sum_{K=1}^{N} Q_{K}^{1}\left(x_{1}\right) Q_{N-K+1}^{1}\left(x_{2}\right) \\
= & Q_{N+1}^{1}\left(x_{1}\right)-Q_{N+1}^{1}\left(x_{2}\right),
\end{aligned}
$$

which is equivalent to eq. (A.5). Now it is straightforward to verify eq. (A.4) for $M>1$, as well. Denoting $y \equiv\left\{x_{3}, \ldots, x_{M+1}\right\}$, we have

$$
\begin{aligned}
&\left(x_{1}-x_{2}\right) Q_{N}^{M+1}\left(x_{1}, x_{2}, y\right) \stackrel{\stackrel{\text { A.3 })}{=}}{=}\left(x_{1}-x_{2}\right) \sum_{K=0}^{N} Q_{K}^{2}\left(x_{1}, x_{2}\right) Q_{N-K}^{M-1}(y) \\
& \stackrel{(\text { A.6) }}{=} \sum_{K=0}^{N}\left(Q_{K+1}^{1}\left(x_{1}\right)-Q_{K+1}^{1}\left(x_{2}\right)\right) Q_{N-K}^{M-1}(y) \\
&=\sum_{K=1}^{N+1}\left(Q_{K}^{1}\left(x_{1}\right)-Q_{K}^{1}\left(x_{2}\right)\right) Q_{N-K+1}^{M-1}(y) \\
& \quad+\left(Q_{0}^{1}\left(x_{1}\right)-Q_{0}^{1}\left(x_{2}\right)\right) Q_{N+1}^{M-1}(y) \\
&=Q_{N+1}^{M}\left(x_{1}, y\right)-Q_{N+1}^{M}\left(x_{2}, y\right)
\end{aligned}
$$

and therefore finishing the proof of the lemma.

\section{A.2 Flavour Expansion Theorem: the proof}

Proof. Due to Def. 1, the Hermitian matrix function $f(\mathbf{A})$, can be expressed as a power series,

$$
f(\mathbf{A})=\sum_{m=0}^{\infty} c_{m} \mathbf{A}^{m} .
$$


One can apply the matrix decomposition $\mathbf{A}=\mathbf{A}_{\mathbf{0}}+\hat{\mathbf{A}}$ to the above series (convergent by assumption) and rearrange terms collecting together the same powers of $\hat{\mathbf{A}}$. Assuming that the resulting summation remains convergent, we have

$$
\begin{aligned}
& f(\mathbf{A})=c_{0} \mathbf{I}+c_{1} \mathbf{A}_{\mathbf{0}}+c_{2} \mathbf{A}_{\mathbf{0}}^{2}+c_{3} \mathbf{A}_{\mathbf{0}}^{3}+\ldots: \mathbf{F}_{\mathbf{0}} \\
& +c_{1} \hat{\mathbf{A}}+c_{2}\left\langle\hat{\mathbf{A}} \mathbf{A}_{\mathbf{0}}\right\rangle+c_{3}\left\langle\hat{\mathbf{A}} \mathbf{A}_{\mathbf{0}}^{2}\right\rangle+c_{4}\left\langle\hat{\mathbf{A}} \mathbf{A}_{\mathbf{0}}^{3}\right\rangle+\ldots: \mathbf{F}_{\mathbf{1}} \\
& +c_{2} \hat{\mathbf{A}}^{2}+c_{3}\left\langle\hat{\mathbf{A}}^{2} \mathbf{A}_{\mathbf{0}}\right\rangle+c_{4}\left\langle\hat{\mathbf{A}}^{2} \mathbf{A}_{\mathbf{0}}^{2}\right\rangle+\ldots: \mathbf{F}_{\mathbf{2}} \\
& \ddots \\
& +c_{M} \hat{\mathbf{A}}^{M}+c_{M+1}\left\langle\hat{\mathbf{A}}^{M} \mathbf{A}_{\mathbf{0}}\right\rangle+\ldots+c_{n}\left\langle\hat{\mathbf{A}}^{M} \mathbf{A}_{\mathbf{0}}^{n-M}\right\rangle+\ldots \quad: \mathbf{F}_{\mathbf{M}} \\
& +c_{M+1} \hat{\mathbf{A}}^{M+1}+\ldots+c_{n}\left\langle\hat{\mathbf{A}}^{M+1} \mathbf{A}_{\mathbf{0}}^{n-M-1}\right\rangle+\ldots: \mathbf{F}_{\mathbf{M}+\mathbf{1}}
\end{aligned}
$$

where we have defined

$$
\left\langle\hat{\mathbf{A}}^{m} \mathbf{A}_{\mathbf{0}}^{n}\right\rangle \equiv \sum_{P-\text { distinct }} \hat{\mathbf{A}}^{m} \mathbf{A}_{\mathbf{0}}^{n},
$$

for all distinct permutations of the set $\left\{\hat{\mathbf{A}} \ldots, \mathbf{A}_{\mathbf{0}} \ldots\right\}$ of $(m+n)$ objects.

The matrix element $\langle I|f(A)| J\rangle$ will be given by the sum

$$
(f(A))^{I J}=\sum_{N=0}^{\infty} F_{N}^{I J}=F_{0}^{I J}+F_{1}^{I J}+F_{2}^{I J}+\ldots,
$$

where, by direct calculation, the above terms are given by (summation over repeated internal indices $K_{i}$, is considered - also if they appear more than twice),

$$
\begin{aligned}
F_{0}^{I J}= & \delta^{I J} f\left(A_{0}^{I}\right) \\
F_{1}^{I J}= & \hat{A}_{I J}\left(c_{1}+c_{2}\left[A_{0}^{I}+A_{0}^{J}\right]+c_{3}\left[\left(A_{0}^{I}\right)^{2}+\left(A_{0}^{J}\right)^{2}+A_{0}^{I} A_{0}^{J}\right]+\ldots\right) \\
F_{2}^{I J}= & \hat{A}_{I K_{1}} \hat{A}_{K_{1} J}\left(c_{2}+c_{3}\left[A_{0}^{I}+A_{0}^{J}+A_{0}^{K_{1}}\right]\right. \\
& \left.+c_{4}\left[\left(A_{0}^{I}\right)^{2}+\left(A_{0}^{J}\right)^{2}+\left(A_{0}^{K_{1}}\right)^{2}+A_{0}^{I} A_{0}^{J}+A_{0}^{I} A_{0}^{K_{1}}+A_{0}^{J} A_{0}^{K_{1}}\right]+\ldots\right) \\
\quad & \hat{A}_{I K_{1}} \hat{A}_{K_{1} J} \sum_{N=0}^{\infty} c_{2+N} Q_{N}^{3}\left(A_{0}^{I}, A_{0}^{J}, A_{0}^{K_{1}}\right) \\
\ldots & \\
F_{M}^{I J}= & \hat{A}_{I K_{1}} \hat{A}_{K_{1} K_{2}} \ldots \hat{A}_{K_{M-1} J} \sum_{N=0}^{\infty} c_{M+N} Q_{N}^{M+1}\left(A_{0}^{I}, A_{0}^{J}, A_{0}^{K_{1}}, \ldots, A_{0}^{K_{M-1}}\right),
\end{aligned}
$$

To prove the theorem, we need to show

$$
\sum_{N=0}^{\infty} c_{M+N} Q_{N}^{M+1}\left(A_{0}^{I}, A_{0}^{J}, A_{0}^{K_{1}}, \ldots, A_{0}^{K_{M-1}}\right)=f^{[M]}\left(A_{0}^{I}, A_{0}^{J}, A_{0}^{K_{1}}, \ldots, A_{0}^{K_{M-1}}\right),
$$


for all $M \geq 0$. This can be realized using mathematical induction. For $M=0$, we obtain trivially

$$
\sum_{N=0}^{\infty} c_{N} Q_{N}^{1}\left(A_{0}^{I}\right)=\sum_{N=0}^{\infty} c_{N}\left(A_{0}^{I}\right)^{N}=f\left(A_{0}^{I}\right) \equiv f^{[0]}\left(A_{0}^{I}\right)
$$

Now, let

$$
\sum_{N=0}^{\infty} c_{M+N} Q_{N}^{M+1}=f^{[M]},
$$

holds for some $M>0$ and for any set of $M+1$ arguments. Then, we need to show that

$$
\sum_{N=0}^{\infty} c_{M+N+1} Q_{N}^{M+2}\left(A_{0}^{I}, A_{0}^{J}, A_{0}^{K_{1}}, \ldots, A_{0}^{K_{M}}\right)=f^{[M+1]}\left(A_{0}^{I}, A_{0}^{J}, A_{0}^{K_{1}}, \ldots, A_{0}^{K_{M}}\right),
$$

which, by Def. 2 of divided differences in eq. (3.7), is equivalent to showing

$$
\begin{aligned}
\left(A_{0}^{I}-A_{0}^{J}\right) \sum_{N=0}^{\infty} c_{M+N+1} Q_{N}^{M+2}\left(A_{0}^{I}, A_{0}^{J}, A_{0}^{K_{1}}, \ldots, A_{0}^{K_{M}}\right) & \\
\stackrel{(\mathrm{A} .4)}{=} & \sum_{N=0}^{\infty} c_{M+N+1}\left(Q_{N+1}^{M+1}\left(A_{0}^{I}, A_{0}^{K_{1}}, \ldots\right)-Q_{N+1}^{M+1}\left(A_{0}^{J}, A_{0}^{K_{1}}, \ldots\right)\right) \\
= & \sum_{N=1}^{\infty} c_{M+N}\left(Q_{N}^{M+1}\left(A_{0}^{I}, A_{0}^{K_{1}}, \ldots\right)-Q_{N}^{M+1}\left(A_{0}^{J}, A_{0}^{K_{1}}, \ldots\right)\right) \\
& +c_{M}\left(Q_{0}^{M+1}\left(A_{0}^{I}, A_{0}^{K_{1}}, \ldots\right)-Q_{0}^{M+1}\left(A_{0}^{J}, A_{0}^{K_{1}}, \ldots\right)\right) \\
= & \sum_{N=0}^{\infty} c_{M+N}\left(Q_{N}^{M+1}\left(A_{0}^{I}, A_{0}^{K_{1}}, \ldots\right)-Q_{N}^{M+1}\left(A_{0}^{J}, A_{0}^{K_{1}}, \ldots\right)\right) \\
= & f^{[M]}\left(A_{0}^{I}, A_{0}^{K_{1}}, \ldots\right)-f^{[M]}\left(A_{0}^{J}, A_{0}^{K_{1}}, \ldots\right),
\end{aligned}
$$

and hence the theorem is proved.

\section{B Convergence criterion for FET expansion of the one-loop functions}

It is well known that, any one-loop amplitude can be expressed as a linear combination of "master" PV-integrals with trivial i.e.,equal to 1, integrand numerator. Thus, it is sufficient to find a convergence criterion for the FET expansion only for master integrals. Below we formulate such a criterion for the most often considered case of loop functions with vanishing external momenta. The same criterion can be applied to coefficients of the expansion of one-loop integrals in terms of external momenta (assuming that they are far from thresholds and momentum expansion can be performed) - such coefficients can be also reduced to combinations of master integrals with vanishing momenta.

For vanishing external momenta master integrals can be expressed as

$$
P V_{0}^{(n)}\left(m_{1}^{2}, \ldots, m_{n}^{2}\right)=-i(4 \pi)^{2} \int \frac{d^{4} p}{(2 \pi)^{4}} \frac{1}{\prod_{j=1}^{n}\left(p^{2}-m_{j}^{2}\right)}=(-1)^{n} \int_{0}^{\infty} \frac{u d u}{\prod_{j=1}^{n}\left(u+m_{j}^{2}\right)}(\text { B. } 1)
$$


where we assume $n \geq 3$ to avoid divergent integrals - considering the estimates for finite ones is sufficient to establish the convergence criterion for FET expansion as it depends only on behaviour of higher order terms.

Eq. (B.1) leads immediately to inequality

$$
\left|P V_{0}^{(n+1)}\left(m_{1}^{2}, \ldots, m_{n}^{2}, m_{n+1}^{2}\right)\right| \leq \frac{1}{m_{n+1}^{2}}\left|P V_{0}^{(n)}\left(m_{1}^{2}, \ldots, m_{n}^{2}\right)\right| .
$$

Applying this inequality, iteratively for higher order terms, to majorize the r.h.s. of eq. (3.14) (in what follows we do not write explicitly any PV-function arguments apart from the ones used in the expansion), we get

$$
\begin{aligned}
\left|\left[P V_{0}^{(n)}(\mathbf{A})\right]_{I J}\right| \leq & \left|\delta_{I J} P V_{0}^{(n)}\left(A_{0}^{I}\right)\right|+\left|P V_{0}^{(n+1)}\left(A_{0}^{I}, A_{0}^{J}\right) \hat{A}_{I J}\right| \\
& +\left|P V_{0}^{(n+2)}\left(A_{0}^{I}, A_{0}^{J}, A_{0}^{K}\right) \hat{A}_{I K} \hat{A}_{K J}\right|+\ldots \\
\leq & \left|\delta_{I J} P V_{0}^{(n)}\left(A_{0}^{I}\right)\right|+\left|P V_{0}^{(n+1)}\left(A_{0}^{I}, A_{0}^{J}\right)\right|\left|\hat{A}_{I J}\right| \\
& \quad+\left|P V_{0}^{(n+2)}\left(A_{0}^{I}, A_{0}^{J}, A_{0}^{K}\right)\right|\left|\hat{A}_{I K}\right|\left|\hat{A}_{K J}\right|+\ldots \\
\leq & \left|P V_{0}^{(n)}\left(A_{0}^{I}\right)\right|\left(\delta_{I J}+\frac{\left|\hat{A}_{I J}\right|}{A_{0}^{J}}+\frac{\left|\hat{A}_{I K}\right|\left|\hat{A}_{K J}\right|}{A_{0}^{K}}+\ldots\right) \\
& =\left|P V_{0}^{(n)}\left(A_{0}^{I}\right)\right|\left(\delta_{I J}+\sqrt{\frac{A_{0}^{I}}{A_{0}^{J}}}\left(\frac{\left|\hat{A}_{I J}\right|}{\sqrt{A_{0}^{I} A_{0}^{J}}}+\frac{\left|\hat{A}_{I K}\right|}{\sqrt{A_{0}^{I} A_{0}^{K K}}} \frac{\left|\hat{A}_{K J}\right|}{\sqrt{A_{0}^{K} A_{0}^{J}}}+\ldots\right)\right),
\end{aligned}
$$

where we assume that all indices apart from $I, J$ are implicitly summed in the range $1 \ldots N$. Let us now define the symmetric matrix $\mathbf{Q}$ with elements being the absolute values of dimensionless quantities commonly referred in the literature as "mass insertions" (diagonal elements of $\mathbf{Q}$ vanish by definition of $\hat{\mathbf{A}}$ matrix)

$$
Q_{I J}=\frac{\left|\hat{A}_{I J}\right|}{\sqrt{A_{0}^{I} A_{0}^{J}}} .
$$

Then eq. (B.4) can be expressed as

$$
\left|\left[P V_{0}^{(n)}(\mathbf{A})\right]_{I J}\right| \leq\left|P V_{0}^{(n)}\left(A_{0}^{I}\right)\right|\left(\delta_{I J}+\sqrt{\frac{A_{0}^{I}}{A_{0}^{J}}}\left(\mathbf{Q}+\mathbf{Q}^{2}+\ldots\right)_{I J}\right) .
$$

The expression in the inner parenthesis of the r.h.s. of eq. (B.5) is a geometric series. According to the definition of a function of Hermitian matrix given in section 3.1, this series is convergent if it converges also for any of $\mathbf{Q}$ eigenvalues, hence their absolute values must be all smaller than 1 . This can be expressed formally, as

$$
\sup _{\|\mathbf{e}\|=1}\left|\mathbf{e}^{\top} \mathbf{Q} \mathbf{e}\right|=\sup _{\|\mathbf{e}\|=1}\left|\mathbf{e}^{\top} \mathbf{D}_{Q} \mathbf{e}\right|<1,
$$

where $\mathbf{e}$ denotes any real unit vector, and $\mathbf{D}_{Q}$ is the diagonal matrix of eigenvalues. Obviously, this is a sufficient but not necessary condition for the convergence of the expansion. 
Finally we should note that vanishing diagonal elements can not pose a threat for the convergence of the FET expansion in physical theories. This is because all Hermitian (squared) mass matrices are semi-positive definite matrices, and for such matrices if $A_{0}^{I}=0$, then necessarily also $\hat{A}_{I K}=\hat{A}_{K I}=0$ for all $K$. Thus all potentially divergent terms vanish.

Open Access. This article is distributed under the terms of the Creative Commons Attribution License (CC-BY 4.0), which permits any use, distribution and reproduction in any medium, provided the original author(s) and source are credited.

\section{References}

[1] S. Weinberg, A model of leptons, Phys. Rev. Lett. 19 (1967) 1264 [INSPIRE].

[2] S. Glashow, Partial symmetries of weak interactions, Nucl. Phys. 22 (1961) 579.

[3] A. Salam, Weak and electromagnetic Interactions in Proceedings of the Eighth Nobel Symposium, N. Svartholm ed., Wiley, New York U.S.A. (1968).

[4] N. Cabibbo, Unitary symmetry and leptonic decays, Phys. Rev. Lett. 10 (1963) 531 [INSPIRE].

[5] M. Kobayashi and T. Maskawa, CP violation in the renormalizable theory of weak interaction, Prog. Theor. Phys. 49 (1973) 652 [InSPIRE].

[6] B. Pontecorvo, Mesonium and anti-mesonium, Sov. Phys. JETP 6 (1957) 429 [InSPIRE].

[7] Z. Maki, M. Nakagawa and S. Sakata, Remarks on the unified model of elementary particles, Prog. Theor. Phys. 28 (1962) 870 [INSPIRE].

[8] F. Gabbiani, E. Gabrielli, A. Masiero and L. Silvestrini, A complete analysis of FCNC and CP constraints in general SUSY extensions of the standard model, Nucl. Phys. B 477 (1996) 321 [hep-ph/9604387] [INSPIRE].

[9] M. Misiak, S. Pokorski and J. Rosiek, Supersymmetry and FCNC effects, Adv. Ser. Direct. High Energy Phys. 15 (1998) 795 [hep-ph/9703442] [INSPIRE].

[10] J. Hisano and D. Nomura, Solar and atmospheric neutrino oscillations and lepton flavor violation in supersymmetric models with the right-handed neutrinos, Phys. Rev. D 59 (1999) 116005 [hep-ph/9810479] [inSPIRE].

[11] R. Bhatia, Matrix analysis, Springer, Germany (1997).

[12] R.A. Horn and C.R. Johnson, Matrix analysis, Cambridge University Press, Cambridge U.K. (1990).

[13] G. Passarino and M.J.G. Veltman, One loop corrections for $e^{+} e^{-}$annihilation into $\mu^{+} \mu^{-}$in the Weinberg model, Nucl. Phys. B 160 (1979) 151 [INSPIRE].

[14] C. de Boor, Divided differences, Surv. Approx. Theory 1 (2005) 46 [math/0502036].

[15] A.J. Buras, A. Romanino and L. Silvestrini, $K \rightarrow \pi$ neutrino anti-neutrino: a model independent analysis and supersymmetry, Nucl. Phys. B 520 (1998) 3 [hep-ph/9712398] [INSPIRE].

[16] G.F. Giudice, M. Nardecchia and A. Romanino, Hierarchical soft terms and flavor physics, Nucl. Phys. B 813 (2009) 156 [arXiv:0812.3610] [InSPIRE].

[17] A. Crivellin and J. Girrbach, Constraining the MSSM sfermion mass matrices with light fermion masses, Phys. Rev. D 81 (2010) 076001 [arXiv:1002.0227] [INSPIRE]. 
[18] A. Dedes, M. Paraskevas, J. Rosiek, K. Suxho and K. Tamvakis, Rare top-quark decays to Higgs boson in MSSM, JHEP 11 (2014) 137 [arXiv:1409.6546] [INSPIRE].

[19] H.P. Nilles, Supersymmetry, supergravity and particle physics, Phys. Rept. 110 (1984) 1 [INSPIRE].

[20] H.E. Haber and G.L. Kane, The search for supersymmetry: probing physics beyond the standard model, Phys. Rept. 117 (1985) 75 [INSPIRE].

[21] S.P. Martin, A supersymmetry primer, Adv. Ser. Direct. High Energy Phys. 21 (2010) 1 [hep-ph/9709356] [INSPIRE].

[22] K. Fuyuto, J. Hisano, N. Nagata and K. Tsumura, QCD corrections to quark (chromo)electric dipole moments in high-scale supersymmetry, JHEP 12 (2013) 010 [arXiv: 1308.6493] [INSPIRE].

[23] A. Manohar and H. Georgi, Chiral quarks and the nonrelativistic quark model, Nucl. Phys. B 234 (1984) 189 [INSPIRE].

[24] S. Pokorski, J. Rosiek and C.A. Savoy, Constraints on phases of supersymmetric flavor conserving couplings, Nucl. Phys. B 570 (2000) 81 [hep-ph/9906206] [INSPIRE].

[25] J. Rosiek, Complete set of Feynman rules for the MSSM: erratum, hep-ph/9511250 [INSPIRE].

[26] J. Rosiek, Complete set of feynman rules for the minimal supersymmetric extension of the standard model, Phys. Rev. D 41 (1990) 3464 [INSPIRE].

[27] C.A. Baker et al., An improved experimental limit on the electric dipole moment of the neutron, Phys. Rev. Lett. 97 (2006) 131801 [hep-ex/0602020] [INSPIRE].

[28] J. Rosiek, P. Chankowski, A. Dedes, S. Jager and P. Tanedo, SUSY_FLAVOR: a computational tool for FCNC and CP-violating processes in the MSSM, Comput. Phys. Commun. 181 (2010) 2180 [arXiv:1003.4260] [INSPIRE].

[29] A. Crivellin et al., SUSY_FLAVOR v2: a computational tool for FCNC and CP-violating processes in the MSSM, Comput. Phys. Commun. 184 (2013) 1004 [arXiv:1203.5023] [INSPIRE].

[30] J. Rosiek, SUSY_FLAVOR library and constraints on $B_{s} \rightarrow \mu^{+} \mu^{-}$decay rate, arXiv: 1212.0032 [INSPIRE].

[31] A. Crivellin and J. Rosiek, SUSY_FLAVOR library for rare decays in the MSSM, PoS (EPS-HEP 2013) 081 [arXiv: 1308.6299] [INSPIRE].

[32] J. Rosiek, SUSY_FLAVOR v2.5: a computational tool for FCNC and CP-violating processes in the MSSM, Comput. Phys. Commun. 188 (2014) 208 [arXiv:1410.0606] [InSPIRE].

[33] W. Fulton and J. Harris, Representation theory: a first course, Graduate Texts in Mathematics/Readings in Mathematics. Springer (1991). 